\title{
SÍNDROME DE BURNOUT EM ATLETAS DE FUTSAL FEMININO U- NIVERSITÁRIAS: UM ESTUDO COMPARATIVO
}

\author{
Thainara Cosvoski \\ Universidade Paranaense, Umuarama, Paraná, Brasil \\ Mateus Dias Antunes \\ Universidade de São Paulo, São Paulo, Brasil \\ Daniel Vicentini de Oliveira \\ União de Faculdades Metropolitanas de Maringá, Maringá, Paraná, Brasil \\ Gabriel Lucas Morais Freire \\ Universidade Federal do Vale do São Francisco, Petrolina, Pernambuco, Brasil \\ José Roberto Andrade do Nascimento Júnior \\ Universidade Federal do Vale do São Francisco, Petrolina, Pernambuco, Brasil \\ Fabio Ricardo Acencio \\ Centro Universitário de Maringá, Maringá, Paraná, Brasil
}

\begin{abstract}
Resumo
Este estudo teve como objetivo comparar os sintomas de burnout de atletas universitárias de futsal feminino em função da região geográfica, ocorrência de lesões e frequência de treinos físicos e táticos. Participaram da pesquisa 114 atletas (20 a 22 anos) que disputaram os Jogos Universitários Brasileiros no ano de 2016. A análise de dados foi conduzida por meio dos testes de Kolmogorov-Smirnov, Friedman, Kruskal-Wallis e "U" de Mann-Whitney ( $<<0,05)$. As atletas de equipes que realizam mais de três treinos táticos durante a semana apresentaram escore significativamente $(\mathrm{p}=0,041)$ superior ao das atletas de equipes que realizam até três treinos. $\mathrm{O}$ volume de treinamentos táticos parece ser um fator interveniente na exaustão física e emocional no contexto do futsal feminino universitário.
\end{abstract}

Palavras-chave: Exaustão emocional. Estresse. Esporte. Universidade.

\section{BURNOUT SYNDROME IN FUTSAL WOMEN'S ATHLETES UNIVERSITY: A COMPARATIVE STUDY}

\begin{abstract}
The objective of this study was to compare the symptoms of burnout in female futsal university athletes as a function of geographical region, occurrence of injuries and frequency of physical and tactical training. A total of 114 athletes (20 to 22 years old) participated in the Brazilian University Games in 2016. Data analysis was conducted using the Kolmogorov-Smirnov, Friedman, Kruskal-Wallis and "U" tests of Mann- Whitney ( $p<0.05)$. Athletes from teams that perform more than three tactical training during the week presented a significantly higher score $(p=0.041)$ than athletes from teams that performed up to three training sessions. The
\end{abstract}


volume of tactical training seems to be an intervening factor in the physical and emotional exhaustion in the context of university female futsal.

Keywords: Emotional exhaustion. Stress. Sport. University.

\title{
SÍNDROME DE BURNOUT EN ATLETAS DE FUTSAL FEMENINO UNIVERSITA- RIAS: UN ESTUDIO COMPARATIVO
}

\begin{abstract}
Resumen
Este estudio tuvo como objetivo comparar los síntomas de burnout de atletas universitarios de futsal femenina en función de la región geográfica, ocurrencia de lesiones y frecuencia de entrenamientos físicos y tácticos. En el año 2016. El análisis de datos fue conducido por medio de las pruebas de Kolmogorov-Smirnov, Friedman, Kruskal-Wallis y "U" de MannWhitney ( $\mathrm{p}<0,05)$. Los atletas de equipos que realizan más de tres entrenamientos tácticos durante la semana presentaron una puntuación significativamente $(\mathrm{p}=0,041)$ superior al de las atletas de equipos que realizan hasta tres entrenamientos. El volumen de entrenamientos tácticos parece ser un factor interviniente en el agotamiento físico y emocional en el contexto del futsal femenino universitario.
\end{abstract}

Palabras clave: Extracción emocional. El estrés. Deporte. Universidad.

\section{Introdução}

Na prática do esporte competitivo, é fundamental que a preparação dos atletas seja oriunda de uma atividade sistemática que vise a promover adaptações morfológicas, metabólicas e funcionais, para que os atletas consigam ter um ótimo desempenho na competição (MOREIRA et al., 2012; FORTES, COSTA \& FERREIRA, 2018). Com isso, essa prática requer do atleta dedicação integral, com treinamentos diários, intensa preparação física e técnica, exigindo uma performance apurada dos atletas, que muitas vezes gera uma sobrecarga física e psíquica (GAYARDO; MATANA; SILVA, 2012).

Essas sobrecargas podem gerar algumas ocorrências psíquicas no atleta, como o estresse pré-competitivo (NASCIMENTO JUNIOR et al., 2011; CAPUTO; ROMBADI \& DA SILVA, 2017) e, em alguns casos, a síndrome de burnout, que tem origem na incompatibilidade dos atletas em lidar com as demandas estressantes do esporte (SOUZA et al, 2018; FORTES, COSTA \& FERREIRA, 2018).

Burnout, por sua vez, diz respeito ao esgotamento físico e mental (SOUZA et al, 2018). Este conjunto multidimensional psicofisiológico de saúde que forma a síndrome é dividida em três dimensões: exaustão física e emocional, reduzido senso de realização esportiva e desvalorização da modalidade esportiva (GUSTAFSSON et al, 2008). Como consequência, atletas com alta magnitude de sintomas de burnout podem ter o seu desempenho esportivo prejudicado ou tomar a decisão de abandonar a prática (FORTES; COSTA \& FERREIRA, 2018).

Uma gama de estudos destaca que atletas de elite praticantes de esportes perfeccionistas como tênis e ginástica são mais sensíveis à síndrome de burnout pelo fato de sofrerem grande influência externa (KANG et al, 2016; MADIGAN, STOEBER \& PASSFIELD, 2016). SCHWELLNUS et al (2016) descrevem como fator de estresse dominante para o desenvolvimento do burnout as práticas inconsistentes dos treinadores, bem como o número de lesões provocadas por essa prática excessiva e mal planejada, devido à natureza repetitiva do esforço físico e cargas excessivas de treinamento. 
Diante disso, o presente estudo teve como objetivo comparar os sintomas de burnout de atletas de futsal feminino participantes dos Jogos Universitários Brasileiros no ano de 2016 em função da região geográfica, ocorrência de lesões e frequência de treinos físicos e táticos.

\section{Método}

\section{Participantes}

Fizeram parte da pesquisa 114 atletas de futsal feminino com idades de 20 a 22 anos que disputaram os Jogos Universitários Brasileiros (JUBS) no ano de 2016. As atletas eram representantes de equipes das regiões sul $(n=26)$, sudeste $(n=16)$, centro-oeste $(n=22)$ e nordeste $(n=50)$. A seleção da amostra foi probabilística aleatória simples e os critérios de inclusão foram os seguintes: 1) ter idade a partir de 18 anos; 2) participar da fase estadual e nacional da competição; e 3) não estar lesionada. Somente participaram do estudo as atletas que assinaram o Termo de Consentimento Livre e Esclarecido e que verbalmente manifestaram o desejo de participar da pesquisa.

\section{Instrumentos}

Foi aplicado um questionário de dados sociodemográficos com questões referentes à idade, à ocorrência de lesão na última temporada, à região geográfica, ao tempo de prática, ao tempo de duração e à frequência semanal de treinamentos físicos e táticos.

Para análise da síndrome de burnout foi utilizado o Questionário de Burnout para Atletas (QBA), elaborado por Raedeke e Smith (2001) e validado para o contexto brasileiro por Pires, Brandão e Silva (2006). O QBA é composto por 15 itens distribuídos por três dimensões: a) a exaustão física e emocional; b) a desvalorização esportiva; e c) o reduzido senso de realização esportiva. Os itens são respondidos por meio de uma escala Likert de cinco pontos em um continuum de "Quase Nunca" (1) a "Quase Sempre" (5). Os resultados são obtidos a partir da média aritmética das respostas dadas aos cinco itens correspondentes a cada dimensão de burnout.

\section{Coleta de dados}

Os procedimentos adotados nesta pesquisa obedeceram aos critérios da Ética em Pesquisa com Seres Humanos, conforme Resolução n ${ }^{\circ}$ 466/12 do Conselho Nacional de Saúde. O estudo foi aprovado pelo Comitê de Ética em Pesquisa sob o Parecer n. 2.412.651. Inicialmente foi realizado o contato com os treinadores das equipes para solicitação da autorização para realização das coletas de dados com as atletas. As coletas foram realizadas no local de treino, meia hora antes do início das atividades. A aplicação dos questionários foi realizada de forma coletiva e o preenchimento dos questionários teve duração de aproximadamente 15 minutos.

\section{Análise de dados}

A análise e a estatística dos dados foram realizadas mediante a utilização do programa estatístico SPSS, versão 25.0. As variáveis qualitativas foram resumidas em frequências absolutas e relativas. Para análise da distribuição dos dados, utilizou-se o teste de KolmogorovSmirnov. Como os dados não apresentaram distribuição normal, foram utilizados Mediana (Md) e Quartis (Q1; Q3) para caracterização dos resultados dos dados numéricos. Para comparar as dimensões de burnout intragrupo, efetuou-se o Teste de Friedman. Para a comparação 
das dimensões de burnout entre os grupos foi utilizado o teste de Kruskal-Wallis e "U" de Mann-Whitney. Foi adotada a significância de $\mathrm{p}<0,05$.

\section{Resultados}

Conforme os resultados da Tabela 1 , nota-se que a dimensão de burnout com maior valor entre as atletas foi o Reduzido senso de realização $(\mathrm{Md}=2,5)$, seguida de Desvalorização esportiva $(M d=2,0)$ e Exaustão física e emocional $(M d=1,0)$. Apesar dessas diferenças, os valores das dimensões de burnout variaram entre "raramente", "raramente e algumas vezes" e "quase nunca".

Tabela 1. Sintomas de burnout das atletas de futsal participantes dos Jogos Universitários Brasileiros (JUBS) no ano de 2016.

\begin{tabular}{lcc}
\hline \multirow{2}{*}{ Dimensões de burnout } & Amostra $(\mathbf{n}=\mathbf{1 1 4})$ & \multirow{2}{*}{$\mathbf{P}$} \\
\cline { 2 - 2 } & \multicolumn{1}{c}{$\mathbf{M d}(\mathbf{Q 1 - Q 3})$} & $0,001^{*}$ \\
Desvalorização esportiva & $2,00(0,00-5,00)^{\mathrm{b}}$ & $0,001^{*}$ \\
Reduzido senso de realização & $2,50(0,00-4,50)^{\mathrm{a}}$ & $0,001^{*}$ \\
Exaustão física e emocional & $1,00(0,00-3,00)^{\mathrm{c}}$ & 0 \\
\hline
\end{tabular}

* Diferença significativa $(\mathrm{p}<0,05)$ - Teste de Friedman seguido do teste de Wilcoxon.

Não foi encontrada diferença significativa $(\mathrm{p}>0,05)$ nas dimensões de burnout das atletas em função da região geográfica de suas equipes (Tabela 2), indicando que a região geográfica não parece ser um elemento interveniente nos sintomas de burnout das atletas universitárias. Todos os grupos apresentaram valores maiores na dimensão de reduzido senso de realização e menor escore na exaustão física e emocional.

Tabela 2. Comparação das dimensões de burnout das atletas de futsal participantes dos Jogos Universitários Brasileiros (JUBS), no ano de 2016, em função da região geográfica da equipe.

\begin{tabular}{lcccc}
\hline \multirow{2}{*}{ Dimensões de burnout } & $\begin{array}{c}\text { Sul } \\
(\mathbf{n = 2 6})\end{array}$ & $\begin{array}{c}\text { Sudeste } \\
(\mathbf{n = 1 6})\end{array}$ & $\begin{array}{c}\text { Centro-oeste } \\
(\mathbf{n = 2 2})\end{array}$ & $\begin{array}{c}\text { Nordeste } \\
(\mathbf{n = 5 0})\end{array}$ \\
\cline { 2 - 5 } & $\mathbf{M d}(\mathbf{Q 1 - Q 3})$ & $\mathbf{M d}(\mathbf{Q 1 - Q 3})$ & $\mathbf{M d}(\mathbf{Q 1 - Q 3})$ & Md (Q1-Q3) \\
\hline Exaustão física e emocio- & $1,72(1,29-$ & $1,79(1,29-$ & $1,50(1,14-$ & $1,64(1,14-$ \\
nal & $2,43)$ & $2,54)$ & $2,21)$ & $2,18)$ \\
Reduzido senso de realiza- & $2,50(2,17-$ & $2,59(2,21-$ & $2,33(2,17-$ & $2,50(2,13-$ \\
ção & $2,83)$ & $2,83)$ & $2,87)$ & $2,87)$ \\
Desvalorização esportiva & $2,00(1,50-$ & $2,00(1,12-$ & $2,25(1,50-$ & $2,00(1,00-$ \\
& $2,63)$ & $3,00)$ & $3,00)$ & $3,00)$ \\
\cline { 2 - 4 } & & &
\end{tabular}

*Diferença significativa $(\mathrm{p}<0,05)$ - Teste de Kruskal-Wallis.

Ao comparar as dimensões de burnout das atletas de futsal participantes dos Jogos Universitários Brasileiros (JUBS) em função da ocorrência de lesão (Tabela 3), não houve diferença significativa entre os grupos, evidenciando que no contexto do futsal universitário feminino a ocorrência de lesões não parece interferir nos sintomas de burnout. 
Tabela 3. Comparação das dimensões de burnout das atletas de futsal participantes dos Jogos Universitários Brasileiros (JUBS) no ano de 2016 em função da ocorrência de lesão.

\begin{tabular}{|c|c|c|c|}
\hline \multirow{3}{*}{ Dimensões de burnout } & \multicolumn{2}{|c|}{ Lesão na última temporada } & \multirow{3}{*}{$\mathbf{P}$} \\
\hline & $\operatorname{Sim}(n=53)$ & Não $(n=61)$ & \\
\hline & Md (Q1-Q3) & Md (Q1-Q3) & \\
\hline Exaustão física e emocional & $1,71(1,29-2,29)$ & $1,57(1,14-2,43)$ & 0,760 \\
\hline Reduzido senso de realização & $2,50(2,17-2,92)$ & $2,50(2,17-2,67)$ & 0,187 \\
\hline Desvalorização esportiva & $2,00(1,00-3,00)$ & $2,00(1,50-3,00)$ & 0,728 \\
\hline
\end{tabular}

*Diferença significativa $(\mathrm{p}<0,05)$ - Teste "U" de Mann-Whitney.

Ao comparar as dimensões de burnout das atletas de futsal participantes dos Jogos Universitários Brasileiros (JUBS) em função da frequência semanal de treinos físicos e táticos (Tabela 4), verificou-se diferença significativa na dimensão de exaustão física e emocional (p $=0,041$ ) em função da frequência de treinos táticos, indicando que as atletas de equipes que realizam mais de três treinos táticos durante a semana $(\mathrm{Md}=1,86)$ apresentaram maior escore do que as atletas de equipes que realizam até três treinos $(\mathrm{Md}=1,57)$. Apesar de tal diferença, destaca-se que ambos os grupos apresentaram baixo escore de exaustão física e emocional.

Tabela 4. Comparação das dimensões de burnout das atletas de futsal participantes dos Jogos Universitários Brasileiros (JUBS) no ano de 2016 em função da ocorrência de lesão.

\begin{tabular}{|c|c|c|c|}
\hline \multirow{3}{*}{ Dimensões de burnout } & \multicolumn{2}{|c|}{ Treino físico semanal } & \multirow{3}{*}{$\mathbf{P}$} \\
\hline & Até $3(n=68)$ & Mais de $3(n=46)$ & \\
\hline & Md (Q1-Q3) & Md (Q1-Q3) & \\
\hline Exaustão física e emocional & $1,57(1,29-2,14)$ & $1,64(1,25-2,43)$ & 0,490 \\
\hline Reduzido senso de realização & $2,50(2,17-2,83)$ & $2,42(2,17-2,83)$ & 0,308 \\
\hline \multirow[t]{4}{*}{ Desvalorização esportiva } & $2,00(1,00-3,00)$ & $2,50(1,50-3,00)$ & 0,322 \\
\hline & \multicolumn{2}{|c|}{ Treino tático semanal } & \\
\hline & Até $3(n=61)$ & Mais de $3(n=53)$ & \\
\hline & Md (Q1-Q3) & Md (Q1-Q3) & \\
\hline Exaustão física e emocional & $1,57(1,14-2,07)$ & $1,86(1,29-2,43)$ & $0,041 *$ \\
\hline Reduzido senso de realização & $2,50(2,08-2,83)$ & $2,50(2,17-2,83)$ & 0,587 \\
\hline Desvalorização esportiva & $2,00(1,25-3,00)$ & $2,00(1,50-3,00)$ & 0,952 \\
\hline
\end{tabular}

\section{Discussão}

O presente estudo obteve médias relativamente baixas, ou seja, as atletas não apresentaram valores severos de burnout. $\mathrm{O}$ estudo vai ao encontro das pesquisas realizadas por BALBIM, NASCIMENTO JUNIOR \& VIEIRA, 2012 et al. (2016); GIACOMINI \& FONSECA (2014) e PIRES, SOUZA \& SOUZA (2015) em modalidades de esporte coletivo.

Ao analisar a dimensão de "exaustão física e emocional" encontramos a menor média reportada, contrariando os resultados reportados por GOODGER et al. (2007) e Rodrigues (2018), que também encontraram baixa média nessa dimensão. Porém, atletas do sexo feminino apresentam índices mais elevados para exaustão física e emocional quando comparadas aos do sexo masculino.

Quando observamos a mesma dimensão, mas agora na função da frequência de treinos táticos/técnicos, as equipes que tiveram média de duas a três vezes por semana apresentaram relação significativa para a dimensão. Isso pode ser explicado, hipoteticamente, pelo fato de os treinadores focarem seus trabalhos mais nas características táticas da modalidade e na defi- 
ciência técnica de suas atletas, deixando de lado os trabalhos físicos (VARGAS, CAPUTO \& SILVA, 2017).

Ao observar a dimensão "desvalorização esportiva", sabe-se que esses valores aumentam no decorrer da temporada, pois o sentimento de desvalorização esportiva se agrava durante a fase de competição (VERARDI et al., 2015). Entretanto, nossos achados mostram que as atletas ainda tinham comprometimento e dedicação ao esporte praticado, levando em consideração que as equipes avaliadas passaram por fases estaduais para assim participar da fase nacional. Diante desse fato, as atletas podem apresentar um aumento na motivação e confiança a fim de buscar maiores níveis de desempenho (BIM et al. 2014).

A dimensão de "Reduzido senso de realização esportiva" obteve o maior índice na pesquisa. Esse resultado está relacionado com a insatisfação das atletas com suas próprias habilidades e à realização esportiva. Como se sabe, o Futsal ainda é considerado uma modalidade amadora, principalmente quando se trata do gênero feminino, segmento em que os investimentos são escassos, há muito preconceito contra as praticantes e o número de competições são extremamente reduzidos (GIUSTI et al. 2014). Um aspecto importante é o fato de atletas com maior experiência serem mais exigentes com sua performance esportiva, até por existir maior cobrança para se obter um melhor desempenho, com isso são mais vulneráveis para o burnout (RODRIGUES, 2018; PASSOS et al., 2015).

Apesar de alguns autores relacionarem a síndrome de burnout à lesão em atletas (BRENNER et al., 2016; MYER et al., 2015), nosso achado não verificou diferença significativa entre a síndrome e a presença de lesão. Entretanto, VERADI et al. (2014) afirmam que o treinador é crucial para identificar os primeiros sintomas da síndrome de burnout antes que ela se instale, para que, a partir daí, sejam traçadas novas estratégias para tornar a prática mais prazerosa para o atleta, buscando assim recuperá-lo antes que consequências mais definitivas ocorram, como o abandono do esporte e/ou a ocorrência de lesões.

Embora nossos achados estejam de acordo com a literatura em achados anteriores em modalidades coletivas (BALBIM, NASCIMENTO JUNIOR \& VIEIRA, 2012; PIRES, SOUZA, \& SOUZA, 2015), este estudo apresenta algumas limitações que devem ser consideradas. A primeira é a descrição das posições/funções da atleta dentro da equipe, que também podem ter influência nos níveis de estresse e na incidência de burnout, bem como a relação entre esportes individuais e coletivos no gênero feminino, fator apontado por Miranda et al. (2012), que observou na sua revisão que não foi encontrado nenhum estudo específico com mulheres, sendo possível notar a falta de estudos nas bases de dados internacionais relacionadas à síndrome, principalmente com atletas brasileiras, no público feminino. Desta forma, há limitações para realizar comparações dos resultados. Com isso, sugere-se pesquisas relacionadas a essas temáticas para consolidar de vez este recorte na literatura. Estudos sobre os fatores relacionados à burnout no esporte feminino são de suma importância para auxiliar os profissionais na forma de intervenção nessa população.

\section{Conclusão}

Concluiu-se através deste estudo que as atletas universitárias participantes do $64^{\circ}$ JUB's não apresentam níveis alarmantes de burnout, assim como outros esportes que não têm tanta visibilidade dentro do país e, apesar de tantas dificuldades, as atletas continuam motivadas e comprometidas com o esporte. Equipes com melhor desempenho obtiveram médias maiores de burnout. Demonstrou-se uma relação significativa entre a quantidade de treinamentos táticos/técnicos e a exaustão física e emocional, mas a dimensão não obteve altos índices. Pesquisas mais aprofundadas deveriam ser realizadas a fim de demonstrar a realidade de cada equipe, assim como estrutura financeira de treinamentos. 


\section{Referências}

BALBIM, G. M.; NASCIMENTO JUNIOR, J. R. A.; VIEIRA, L. F. RBCDH - Rev. bras. cineantropometria desemp. hum., v. 14, n. 6, p. 704-712, 2012.

BIM, R. H.; NASCIMENTO JUNIOR, J. R. A.; AMORIM, A.; VIEIRA, J. L. L.; VIEIRA, L. F. Estratégias de coping e sintomas de burnout em atletas de futsal de alto rendimento. Revista Brasileira de Ciência e Movimento, v. 22, n. 3, p. 69-75, 2014.

BRENNER, J. S. et al. Sports Specialization and Intensive Training in Young Athletes. Pediatrics. v. 138, n. 3, 2016.

CAPUTO, E. L.; ROMBALDI, A. J.; SILVA, M. C. Sintomas de estresse pré-competitivo em atletas adolescentes de handebol. Revista Brasileira de Ciências do Esporte, v. 39, n. 1, p. 68-72, 2017.

CARVALHO, T.; MIYAZAKI, M. C. O. S. Burnout e enfrentamento em jogadores de futebol: fases pré e durante competição. Revista Brasileira de Medicina do Esporte, v. 20, p. 272-275, 2014.

FORTES, L. S.; COSTA, B. D. V.; FERREIRA, M. E. C. A restrição alimentar e os sintomas de bulimia estão relacionados aos sentimentos de burnout em atletas de esportes coletivos? Revista Brasileira de Ciência e Movimento, v. 26, n. 2, p. 12-17, 2018.

FREUDENBERGER, H. J. Staff Burnout. Journal of social issues, v. 30, n. 1, p. 159-165, 1974.

FREUDENBERGER, H. J. The staff burn-out syndrome in alternative institutions. Psychotherapy: Theory, Research \& Practice, v. 12, n. 1, p. 73, 1975.

FONSECA, G. M.; ZECHIN, F.; MANGINI, R. E. O abandono do futsal na iniciação esportiva. RBFF - Revista Brasileira de Futsal e Futebol, v. 6, n. 21, 2014.

GAYARDO, A.; MATANA, S. B.; SILVA, M. R.. Prevalência de lesões em atletas do futsal feminino brasileiro: um estudo retrospectivo. Revista Brasileira de Medicina do Esporte, v. 18, n. 3, p. 186-9, 2012.

GIUSTI, M. L. Caracterização e perfil motivacional das atletas de futsal feminino de alto rendimento no Brasil. Dissertação (Mestrado profissional em saúde da mulher, criança e adolescente). Universidade Católica de Pelotas, Rio Grande do Sul, 2014.

GIL, R. B.; MONTERO, F. O.; FAYOS, E. J. G.; GULLÓN, J. M. L.; PINTO, A. Otimismo, burnout e estados de humor em desportos de competição. Análise Psicológica, v. 33, n. 2, p. 221-233, 2015.

GOODGER, K.; GORELY, T.; LAVALLEE, D.; HARWOOD, C. H. Burnout in sport: A systematic review. The sport psychologist, v. 21, n. 2, p. 127-151, 2007.

GUSTAFSSON, H.; HASSMÉN, P.; KENTTA, G.; JOHANSSON, M. A qualitative analysis of burnout in elite Swedish athletes. Psychology of sport and exercise, v. 9, n. 6, p. 800-816, 2008.

KANG, K. D. et al. Perfectionism and burnout in women professional golfers. The Journal of sports medicine and physical fitness, v. 56, n. 9, p. 1077-1085, 2016.

MADIGAN, D. J.; STOEBER, J.; PASSFIELD, L. Motivation mediates the perfectionism-burnout relationship: A three-wave longitudinal study with junior athletes. Journal of sport and exercise psychology, v. 38, n. 4, p. 341-354, 2016. 
MIRANDA, V. P. N. et al. Síndrome de burnout e esporte: uma revisão bibliográfica sistêmica. EE Deportes, n. 173, Buenos Aires, 2012.

MOREIRA, A.; MCGUIGAN, M. R.; ARRUDA, A. F.; FREITAS, C. G.; AOKI, M. S. Monitoring internal load parameters during simulated and official basketball matches. The Journal of Strength \& Conditioning Research, v. 26, n. 3, p. 861-866, 2012.

NASCIMENTO JUNIOR, J. R. A. et al. Análise do estresse psicológico pré-competitivo e estratégias de coping de jovens atletas de futebol de campo. Revista Brasileira de Ciência e Movimento, v. 18, n. 4, p. 45-53, 2011.

PASSOS, P. C. B. et al. SATISFAÇÃO DO ATLETA DE FUTSAL E O TEMPO DE EXPERIÊNCIA NO ESPORTE. Revista Brasileira de Ciência e Movimento, v. 23, n. 3, p. 64-69, 2015.

PIRES, D. A. et al. A síndrome de burnout no esporte brasileiro. Journal of Physical Education, v. 23, n. 1, p. 131-139, 2012.

PIRES, D. A. et al. Incidência de burnout em tenistas infantojuvenis. In: Anais do XVIII Congresso Brasileiro de Ciências do Esporte (Conbrace) e V Congresso Internacional de Ciências do Esporte (Conice), Brasília-DF. 2013.

PIRES, D. A.; SOUZA, F. J. S.; DE SOUZA, G. N.. Indicadores das dimensões de burnout nas categorias de base do futebol amador. Caderno de Educação Física e Esporte, v. 13, n. 1, p. 13-19.

PIRES, D. A.; BRANDÃO, M. R. F.; SILVA, C. B. Validação do questionário de burnout para atletas. Journal of Physical Education, v. 17, n. 1, p. 27-36, 2006.

RAEDEKE, T. D.; SMITH, A. L. Development and preliminary validation of an athlete burnout measure. Journal of sport and exercise psychology, v. 23, n. 4, p. 281-306, 2001.

RODRIGUES, T. A. Fatores motivacionais em atletas participantes da modalidade de futsal nos jogos universitários gaúchos de 2014. 32 f. Monografia - Educação Física. Universidade Federal do Rio Grande do Sul, 2014.

RODRIGUES, R. N. Síndrome de Burnout em jovens atletas: um estudo com modalidades esportivas individuais e coletivas na fase pré-competitiva. Dissertação (Mestrado acadêmico em Psicologia do Desenvolvimento e Aprendizagem). Unesp/SP, 2018.

SCHWELLNUS, M. et al. How much is too much? (Part 2) - International Olympic Committee consensus statement on load in sport and risk of illness. British Journal of Sports Medicine. v. 50, n. 17, p. 1043-1052, 2016.

SILVA, S. R. et al. Nível de conhecimento tático e perfeccionismo no futsal. Revista Brasileira de Ciências do Esporte, v. 36, 2016.

SOUZA, R. O. et al. Analysis of Dehydroepiandrosterone Sulphate, Cortisol and Testosterone Levels in Performance Athletes Affected by Burnout Syndrome. Journal of Exercise Physiology Online, v. 21, n. 2, p. 150-157, 2018.

SOUZA, R. O. et al. Analysis of Dehydroepiandrosterone Sulphate, Cortisol and Testosterone Levels in Performance Athletes Affected by Burnout Syndrome. Journal of Exercise Physiology Online, v. 21, n. 2, p. 150-157, 2018. 
VARGAS, L. F.; CAPUTO, E. L.; SILVA, M. C. Caracterização do perfil dos treinadores do futsal feminino de equipes que disputam os jogos abertos de Perola. Rev. Bra. Futsal e Futebol, v. 9, n. 33, p. 151-159, 2017.

VERARDI, C. E. L. et al. Burnout and pre-competition: a study of its occurrence in brazilian soccer players. Revista de Psicología del Deporte, v. 24, p. 259-264, 2015.

VERARDI, C. E. L. et al. Burnout e enfrentamento em jogadores de futebol: fases pré e durante competição. Revista Brasileira de Medicina do Esporte, v. 20, p. 272-275, 2014.

Recebido em: 18/03/2018

Revisado em: 27/10/2018

Aprovado em: 26/11/2018

Endereço para correspondência:

mateus_antunes03@hotmail.com

Mateus Dias Antunes

Universidade de São Paulo

Faculdade de Medicina da Universidade de São Paulo.

Rua Cipotânea

Vila Butantã

05360160 - São Paulo, SP - Brasil 